\title{
Are we winning? Improving perinatal outcomes at a deeply rural district hospital in South Africa
}

\author{
C Benjamin Gaunt
}

Zithulele Hospital is a deeply rural district hospital in the Eastern Cape province. Beset by staffing, procurement and infrastructure problems, the maternity service in 2005 was poorly organised and offered an inadequate standard of care. By our estimates, nearly $50 \%$ of women were still delivering at home. Three years of effort focused on regular auditing to identify problems and improve our service started to bear fruit in 2008, reflected in significantly increased deliveries and a sharp drop in the perinatal mortality rate. We consider some of the successes, which factors have contributed to them, and some remaining challenges.

S Afr Med J 2010; 100: 101-104.
Zithulele Hospital is a deeply rural district hospital in Eastern Cape Province. It receives referrals from the surrounding primary health care clinics and provides non-specialist hospital services to a population of 130000 people living in an area of nearly $1000 \mathrm{~km}^{2}$. The area is one of the poorest in the country, ${ }^{1}$ with low education levels ${ }^{2}$ and very high rates of unemployment. ${ }^{3,4}$ Access to the hospital is by a dirt road not reflected on standard maps.

Founded in 1956 by missionaries, the hospital is now run by the provincial Department of Health. The hospital has struggled to attract health professionals, but this dearth has improved markedly since the arrival of new senior staff in 2005 and 2006; ${ }^{5}$ the multi-disciplinary clinical team now totals 22 professionals.

District rural hospitals in South Africa are historically under-resourced, ${ }^{6}$ the doctor:population ratio is below 18.5 per 100000 in the four most rural provinces. ${ }^{7}$ Standards of maternity care vary widely across the country, but district hospitals typically have worse rates of perinatal and maternal mortality. ${ }^{8}$ With $42 \%$ of deliveries in South Africa conducted at district hospitals, improving their outcomes is an urgent priority. ${ }^{9}$

In Eastern Cape Province, data suggest that $73 \%$ of women have a skilled attendant at delivery. ${ }^{10}$ In 2005 we estimated, on the basis of national fertility rates, the population we serve and deliveries at our hospital, that up to $50 \%$ of women in our area delivered at home.

In addition, the Zithulele maternity service in 2005 was fragmented and disorganised. The labour room was too small, with only two beds, nearly touching each other; there

Zithulele Hospital, Zithulele Village, Mqanduli District, Eastern Cape C Benjamin Gaunt, MB ChB, MSc (PHC), DA (SA), DipObst (SA)

Corresponding author: C Gaunt (gaunts@gmail.com) were only two delivery packs in the hospital, preventing steam sterilisation between cases; the hospital had only one laryngoscope; and other resuscitation equipment was hopelessly inadequate. Poor practices included inadequate monitoring during labour, with no routine use of the partogram, and the use of unrefrigerated ergometrine in the third stage of labour.

Our initial attention was taken up with running day-today services, but our improvement strategy focused on areas of most morbidity and mortality, i.e. HIV, tuberculosis, and maternal and child health.

\section{Methods}

We emphasised the collecting of prospective data, with attention to accuracy and detail, regarding women who deliver in our maternity service. A data sheet was also completed at the time of delivery or as soon as possible thereafter for each perinatal death. These data were entered into the Perinatal Problem Identification Programme (PPIP) version 2.2.3, an audit tool used by 254 institutions in South Africa, and which is managed by the Medical Research Council. ${ }^{11}$ The programme allows the user to analyse basic data and identify avoidable factors associated with each perinatal death.

Use of the PPIP was supplemented with monthly perinatal mortality meetings at which each month's data were presented and every perinatal death discussed. Meetings usually include a short discussion about an obstetric clinical topic and preventive or pre-emptive factors.

We implemented several other significant changes over this period. Training and audit activities were extended. We were the first district hospital in the province to implement the Child Health Problem Identification Programme; this helps us to audit care in the paediatric ward, and drew attention to the importance of the proper implementation of prevention of mother-to-child transmission (PMTCT) strategies for HIV. We have an accredited journal club for doctors to stay up to date and review practice issues, including obstetric care. A system 


\section{Original Articles}

of outreach to four of our feeder clinics allowed input into primary level antenatal care.

Insistence on safe practice was a key intervention. Midwives were instructed to use the partogram for every labour case, and taught how to do this accurately. Use of a cardiotocograph has become routine in all high-risk cases. Protocols were developed for the safe augmentation and induction of labour. Perhaps most importantly, midwives were encouraged to call for help early, especially in the second stage of labour, allowing problems to be dealt with pro-actively. Early summonsing results in more normal labours being seen by the doctor, but readiness to come is important in encouraging midwives to ask for assistance.

\section{Results}

A $53.4 \%$ increase in the number of deliveries at the hospital occurred between 2005 and 2008 - from 745 deliveries in 2005 to 1143 in 2008. A 24-hour caesarean section (CS) service was re-instituted in July 2005, resulting in far fewer referrals to the tertiary hospital $100 \mathrm{~km}$ away, which the patients greatly appreciate.

Despite the increase in deliveries, we achieved a reduction in the 6-monthly perinatal mortality rate. Fig. 1 shows the corrected perinatal mortality rate, but the uncorrected rate for 2008 was also only 27.1 per 1000 . The perinatal care index (PCI), a marker of the quality of care of newborns that corrects for low birth-weight infants, declined from a very high 3.7 during 2006 and 2007 to 2.4 in 2008 . The national average for rural institutions contributing to the PPIP database in 2003 2005 was $2.3 .^{8}$

The perinatal death profile has also changed (Table I). While intrapartum asphyxia remains the predominant cause of death, and the order of causes remains almost the same, the proportion of deaths owing to hypertension, antepartum haemorrhage and infection has decreased.

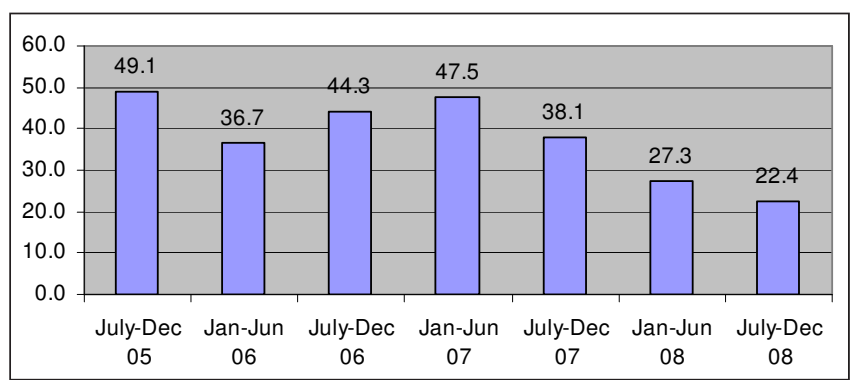

Fig. 1. Corrected perinatal mortality rate over time.

Avoidable factors associated with perinatal death over this period show clearly that accessing care timeously is a major problem (Figs 2 and 3). Lack of transport from home to hospital and delay in presentation during labour are both invariably the consequences of third-world income and infrastructure - a trip to hospital over a weekend can cost up to two-thirds of monthly household income. Problems with antenatal care are the other apparent major issues.

Delivery methods have remained similar over time. The CS rate has remained within a $10-15 \%$ range, rising slightly from $10.5 \%$ in $2005 / 2006$ to $12.3 \%$ in $2007 / 2008$. Assisted vaginal deliveries (which were all ventouse deliveries) rose marginally from $5.4 \%$ to $5.8 \%$ during the same period. One per cent of babies are born as vaginal breech deliveries.

A major success has been in encouraging pregnant women to test for HIV (Fig. 4). Phases in this achievement have included: ensuring that women who deliver in our unit are offered testing, encouraging the clinics to test women antenatally, and introducing dual PMTCT (AZT from 28 weeks and single-dose nevirapine in labour) ahead of the provincial roll-out, having obtained permission from the authorities. In $2008,99.8 \%$ of women who delivered at Zithulele knew their HIV status at delivery.

The seroprevalence rate of HIV has remained $21 \%$ since sufficient data have been collected. This figure compares

\section{Table I. Causes of perinatal death}

\begin{tabular}{lcc}
\hline & & $N(\%)$ \\
\cline { 2 - 3 } & July $2005-$ Dec 2006 & Jan 2007 - Dec 2008 \\
\hline Intrapartum asphyxia & $21(32.8)$ & $32(40)$ \\
Unexplained IUD & $10(15.6)$ & $12(15)$ \\
Spontaneous preterm labour & $8(12.5)$ & $10(12.5)$ \\
APH & $8(12.5)$ & $6(7.5)$ \\
Hypertension & $7(10.9)$ & $5(6.25)$ \\
Infection & $5(7.8)$ & $4(5)$ \\
Fetal abnormality & $0(0)$ & $4(5)$ \\
No obstetric cause & $3(4.7)$ & $3(3.75)$ \\
IUGR & $1(1.6)$ & $2(2.5)$ \\
Trauma & $1(1.6)$ & $1(1.25)$ \\
Other & $0(0)$ & $1(1.25)$ \\
$\quad$ Total & $64(100)$ & $80(100)$ \\
IUD = intra-uterine death; APH = antepartum haemorrhage; IUGR = intra-uterine growth restriction. & \\
\hline
\end{tabular}




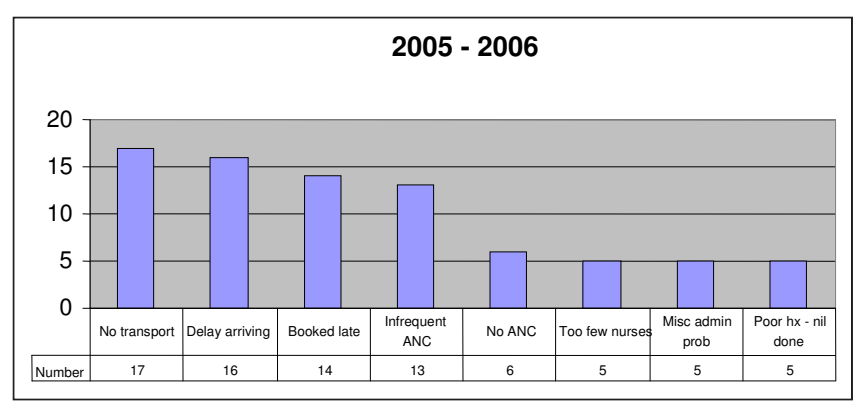

Fig. 2. Foremost 8 avoidable factors in perinatal deaths, July 2005 December 2006.

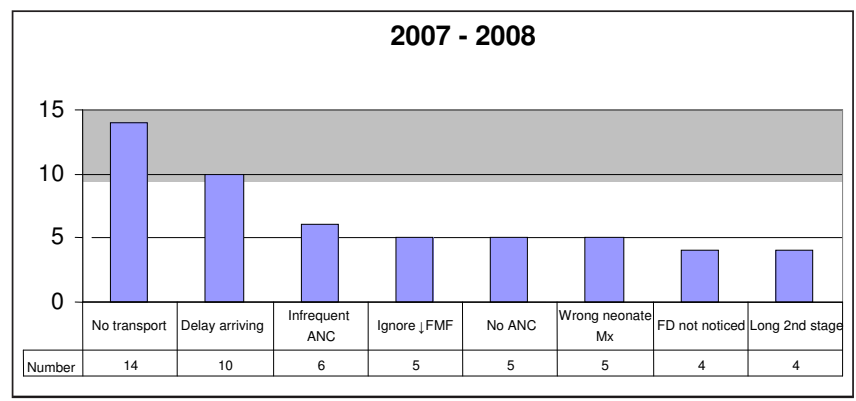

Fig. 3. Foremost 8 avoidable factors in perinatal deaths, January 2007 December 2008.

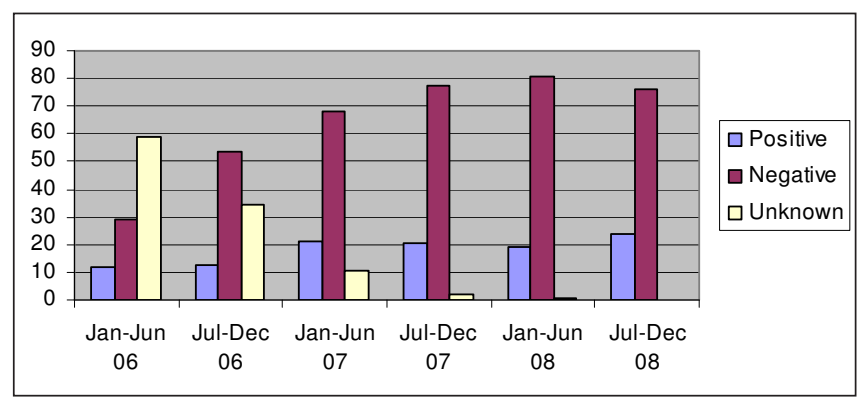

Fig. 4. HIV status at delivery.

favourably with the 2007 rate of $27.3 \%$ in antenatal surveys from the entire OR Tambo District. ${ }^{12}$

A dedicated weekly ARV clinic for pregnant women and children has improved the uptake of PMTCT and HAART in pregnancy; a recent slight rise in the HIV positivity rate is probably owing to us encouraging clinics to refer HIV-positive women to us - the only local site able to offer these services.

\section{Discussion}

It is worthwhile to reflect on the other possible reasons for our success, in addition to the measures we implemented; this assists in identifying the factors justifying continued effort, and may encourage other hospitals to introduce positive changes and assist policymakers aiming to improve district health services overall. This approach is especially relevant as the use of an audit tool such as PPIP does not automatically result in improved perinatal outcomes. ${ }^{13}$
As the ability of women to access care has changed little during this study, we ascribe the increase in attendance to the hospital's improved reputation within the community, as a result of better-quality care.

Despite the improved perinatal mortality rate that we achieved, the PCI remains high. This is partly due to a lowbirth-weight rate of only $10.7 \%$ (compared with a national rural average of $13.3 \%$ ).

The high number of cases of intrapartum asphyxia reflects the poor infrastructure in the area combined with extreme poverty. It also represents a need for continued improvement and heightened vigilance among attending staff.

The avoidable factors related to antenatal care may be created by a combination of the problems of access and a lack of appreciation within the community for the importance of antenatal care, particularly within the context of historically poor service delivery. It is to be hoped that the small improvement over the past 2 years is a sign that conditions are changing as clinics become better staffed and the hospital offers a better service.

A core of skilled staff with long-term commitment to the hospital has undoubtedly provided a foundation for improvements. The four senior staff each have two postgraduate diplomas in different disciplines: two diplomas in obstetrics, two in child health, three in anaesthetics and one in HIV management. Supplemented by enthusiastic community service doctors and local and overseas doctors on one-year contracts or shorter, services have been expanded and improvements sustained, in line with the government's intentions for community service. ${ }^{14}$ Our experience suggests that attracting and retaining long-term staff in rural areas must be more actively prioritised to improve services and provide a better experience for community service doctors. ${ }^{15}$

The cultural theory of organisational change makes three assumptions: that such a thing as organisational culture exists; that culture is related to performance; and that culture can be changed to influence performance. ${ }^{16}$ We are fortunate that much of the ethos of the hospital's founding missionaries has been maintained and that we have an enthusiastic management team. We can affirm the dedicated nursing staff's genuine care for their patients, and have been able to build on this, promoting a culture of excellent, continuously improving standards within the hospital. Our commitment to going the extra mile is evident in our 24-hour CS service which in 2006 was maintained despite only two doctors being present at the hospital for a total of 12 weeks in two separate periods. The CS rate is within the range recommended by the World Health Organization and considered appropriate in our setting. ${ }^{17}$

The introduction of simple protocols, from blood tests on admission to the management of eclampsia, allowed us to standardise the management of conditions commonly encountered. These protocols have been expanded into a 


\section{Original Articles}

handbook that includes orientation information to make it easier for staff to perform their duties despite remaining obstacles. Several pro formas, including a prescription chart for PMTCT and a discharge form, facilitate completing these tasks and are less prone to errors and omissions.

Regarding facilities, our hospital has been part of the government's hospital revitalisation programme, benefiting from new acute wards opened in December 2005. Good facilities lend themselves to being kept clean. The provincial Saving Mothers Saving Babies programme has also provided support for obtaining curtains, linen, patient clothing, medical equipment, computers and extra staff. The overall result was summed up by an opposition parliamentarian describing it as 'a little piece of heaven' during a portfolio committee visit.

New equipment (some donated by the Japanese government) has included an ultrasound machine, proper resuscitation stations, incubators and laryngoscopes. These devices make it possible to aspire to a higher level of care. Policy-makers should not underestimate the role of up-to-date equipment in staff morale and the provision of quality care. ${ }^{18}$ Similarly, the role of a pharmacy, staffed with a pharmacist for the first time, has ensured that there is a good stock of drugs and other supplies.

\section{Conclusion}

The staff at Zithulele Hospital are working hard to improve maternity services in this deeply rural location. We can celebrate significant achievements, including a large increase in deliveries, a decrease in the perinatal mortality rate, an improving profile of the causes of perinatal deaths, and vastly better PMTCT services. Our figures may yet fluctuate because of a variety of factors, and there is still a long way to go, but we hope that, by sharing some of our successes and challenges, we can inspire others in rural settings to make maternal health and improved perinatal care in their areas a priority. We hope our urban colleagues will benefit too, by gaining insight into the challenges faced by their referring district hospitals.

References

1. Health Systems Trust. Health statistics using data from Bradshaw D, Steyn K, eds. Poverty and Chronic Diseases in South Africa: Technical Report 2001. Cape Town: Medical Research Council, 2002. http://www.hst.org.za/healthstats/146/data (accessed 19 May 2008).

2. Statistics South Africa. Census 2001: Census in Brief. Pretoria: Statistics South Africa, 2003 http:// www.statssa.gov.za/census01/html/C2001CensusBrief.asp (accessed 24 October 2006).

3. South African Institute of Race Relations. SAIRR Today: Unemployment and Poverty - An Overview - 28th November 2008. Johannesburg: South African Institute of Race Relations, 2008 http://www.sairr.org.za/sairr-today/news_item.2008-11-28.9488661622 (accessed 10 January 2009)

4. The Oliver Tambo District. Bisho: Eastern Cape Provincial Government. http://www.ecprov. gov.za/page.php?index=13 (accessed 10 January 2009).

5. Bateman C. Multidisciplinary teams - the rural way forward. S Afr Med J 2008; 98(1): 19-22. http://www.samj.org.za/index.php/samj/article/viewFile/860/399 (accessed 1 April 2009).

6. Kautzky K, Tollman S. A perspective on primary health care in South Africa. In: Barron P, ed. Kauth African Health Review 2008. Durban: Health Systems Trust, 2009. http:/ / www.hst.org.
South zou/publications /841 (accessed 1 . Durban: Heal

7. Health Systems Trust. Medical practitioners per 100 000. Durban: Health Systems Trust, 2006 http://www.hst.org.za/healthstats/15/data (accessed 1 April 2009)

8. Pattinson RC, ed. Saving Babies 2003-5: Fifth perinatal care survey of South Africa. Pretoria: Government Printer, 2007

9. Pattinson RC. District Hospitals: The core of maternity services but sadly neglected. In: Abstract Book of the 28th Conference on Priorities in Perinatal Care in Southern Africa, Champagne Sports Resort, 10-13 March 2009

10. World Health Organization. Making Pregnancy Safer: South Africa Country Profile. Geneva: World Health Organization. http://www.who.int/making_pregnancy_safer/events/2008/ mdg5/countries/Final_CP_SouthAfrica_18_09_08.pdf (accessed 1 April 2009).

11. Perinatal Problem Identification Programme, version 2.2.3. Simply Software. www.ppip.co.za.

12. National Department of Health. The National HIV and Syphilis Prevalence Survey: South Africa: 2007. Pretoria: Government Printer, 2008.

13. Pattinson RC, Prinsloo RV. Longitudinal perinatal mortality rates and perinatal care indexes in South Africa. In: Abstract Book of the 28th Conference on Priorities in Perinatal Care in Southern Africa, Champagne Sports Resort, 10 - 13 March 2009.

14. Reid S, Conco D. Monitoring the Implementation of community service. In: Crisp N, ed. South African Health Review 1999. Durban: Health Systems Trust, 2000. http:// www. healthlink.org.za/uploads/files/chapter17_99.pdf (accessed 1 April 2009).

15. De Villiers MR, De Villiers PJT. Doctors' views of working conditions in rural hospitals in the Western Cape. SA Fam Pract 2004; 46(3): 21-26.

16. Scott T, Mannion R, Marshall M, Davies H. Does organisational culture influence health care performance? A review of the evidence. J Health Serv Res Policy 2003; 8(2): 105-117.

17. Ronsmans C, Holtz S, Stanton C. Socioeconomic differentials in caesarean rates in developing countries: a retrospective analysis. Lancet 2006; 368 (9546): 1516-1523.

18. van Holdt K, Murphy M. An Investigation into the Management of Public Hospitals in South Africa: Stressed Institutions, Disempowered Management. Pretoria: Department of Public Service Administration, 2005. http://www.naledi.org.za/pubs/2006/DPSA_research_report_2006. pdf (accessed 1 April 2009.)

Accepted 9 September 2009. 